\title{
Correction to: Nomogram-based estimate of axillary nodal involvement in ACOSOG Z0011 (Alliance): validation and association with radiation protocol variations
}

\author{
Matthew S. Katz ${ }^{1}$ (D $\cdot$ Linda McCall $^{2} \cdot$ Karla Ballman $^{3} \cdot$ Reshma Jagsi $^{4} \cdot$ Bruce G. Haffty $^{5} \cdot$ Armando E. Giuliano $^{6}$
}

Published online: 7 December 2020

○) Springer Science+Business Media, LLC, part of Springer Nature 2020

\section{Correction to: \\ Breast Cancer Research and Treatment \\ (2020) 180:429-436 \\ https://doi.org/10.1007/s10549-020-05555-z}

Unfortunately, the original version of the article was published with incorrect NCT number (NCT00003854) in abstract section".

However, the correct NCT number should read as "NCT00003855". The original article has been corrected.

Publisher's Note Springer Nature remains neutral with regard to jurisdictional claims in published maps and institutional affiliations.

The original article can be found online at https://doi.org/10.1007/ s10549-020-05555-z.

\section{Matthew S. Katz}

Matthew.Katz@lowellgeneral.org

1 Department of Radiation Medicine, Lowell General Hospital, 295 Varnum Avenue, Lowell, MA 01854, USA

2 Alliance Statistics and Data Center, Duke University, Durham, NC, USA

3 Alliance Statistics and Data Center, Weill Cornell Medicine, New York, NY, USA

4 Department of Radiation Oncology, University of Michigan, Ann Arbor, MI, USA

5 Department of Radiation Oncology, Rutgers Cancer Institute of New Jersey, New Brunswick, NJ, USA

6 Department of Surgery, Cedars-Sinai Medical Center, Los Angeles, CA, USA 